\title{
MANGANESE DEPOSITION IN BASAL GANGLIA STRUCTURES RESULTS FROM BOTH PORTAL- SYSTEMIC SHUNTING AND LIVER DYSFUNCTION
}

\section{Christopher Rose*, Roger F. Butterworth*, Joseph Zayed‡, Louise Normandin‡, Kathryn Todd*, Adrianna Michalak*, Laurent Spahr§, Pierre-Michel Huet\$, Gilles Pomier-Layrargues $\S$}

$\S$ Liver Unit * Neuroscience Research Unit, Centre Hospitalier de l'Université de Montréal, Campus Saint-Luc ₹ Department of Environmental and Occupational Health, Université de Montréal, Québec, Canada

\section{ABSTRACT}

Background \& Aims: Manganese (Mn) deposition could be responsible for the $\mathrm{T}_{1}$-weighted magnetic resonance signal hyperintensities observed in cirrhotic patients. These experiments were designed to assess the regional specificity of the Mn increases as well as their relationship to portal-systemic shunting or hepatobiliary dysfunction. Methods: Mn concentrations were measured in (1) brain samples from basal ganglia structures (pallidum, putamen, caudate nucleus) and cerebral cortical structures (frontal, occipital cortex) obtained at autopsy from 12 cirrhotic patients who died in hepatic coma and from 12 matched controls; and from (2) brain samples (caudate/putamen, globus pallidus, frontal cortex) from groups $(n=8)$ of rats either with end-to-side portacaval anastomosis, with biliary cirrhosis, or with fulminant hepatic failure as well as from sham-operated and normal rats. Results: Mn content was significantly increased in frontal cortex (by 38\%), occipital cortex (by 55\%), pallidum (by 186\%), putamen (by 66\%), and caudate (by 54\%) of cirrhotic patients compared with controls. Brain Mn content did not correlate with patient age, etiology of cirrhosis, or history of chronic hepatic encephalopathy. In cirrhotic and portacaval-shunted rats, Mn content was increased in pallidum (by $27 \%$ and $57 \%$, respectively) and in caudate/putamen (by 57\% and 67\%, respectively) compared with control groups. Mn concentration in pallidum was significantly higher in portacaval-shunted rats than in cirrhotic rats. No significant changes in brain Mn concentrations were observed in rats with acute liver failure. Conclusions: These findings suggest that brain $\mathrm{Mn}$ deposition results both from portal-systemic shunting and from liver dysfunction.

Abbreviations MR, magnetic resonance; MRI , MR imaging

INTRODUCTION

Pallidal signal hyperintensity on magnetic resonance imaging (MRI) has been observed in most cirrhotic patients. 1 Increasing evidence suggests that accumulation of manganese could best explain this finding. Pallidal samples obtained at autopsy from cirrhotic patients who died in hepatic encephalopathy contain up to 7 -fold increased

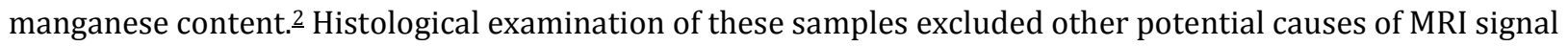
hyperintensities. Furthermore, similar pallidal images have been observed in patients receiving manganese as part of total parenteral nutrition, and hyperintensities disappeared after cessation of manganese administration. $\underline{\underline{3}}$ and $\underline{4}$

We previously reported significant positive correlations between blood manganese, pallidal index (a measure of the degree of magnetic resonance [MR] signal hyperintensity), and the presence of portal-systemic shunting in cirrhotic

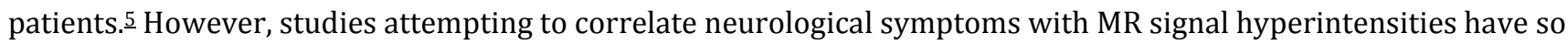
far yielded conflicting results. $\underline{6}, \underline{6}, \underline{g}$ and $\underline{9}$ These inconsistencies could result from the large variability in patient

The final publication is available at http://dx.doi.org/10.1016/S0016-5085(99)70457-9 
Rose, C. et al., 1999. Manganese deposition in basal ganglia structures results from both portal-systemic shunting and liver dysfunction. Gastroenterology, 117(3), p.640-644.

population as well as the different methods of evaluation of neuropsychological status. The aims of the present series of experiments were (1) to measure the regional selectivity of manganese accumulation in the brains of cirrhotic patients compared with appropriate material from matched-controls; and (2) to determine the relative influence of portal-systemic shunting and cholestasis (both factors being suggested as major determinants for brain manganese deposition) on manganese accumulation in the brains of rats with chronic liver failure.

\section{MATERIALS AND METHODS}

\section{Human studies}

Brain samples were obtained from 12 cirrhotic patients who died in hepatic coma and 12 control subjects who had no hepatic, neurological, or psychiatric disorders at the time of death and were matched for age, sex, and autopsy delay times. In no cases had patients or control subjects received total parenteral nutrition before death. Brain dissection was performed using a ceramic knife according to a standardized dissection protocol..10 Brain samples were frozen at $-80^{\circ} \mathrm{C}$ until time of assay. Manganese was measured in the following brain regions: frontal cortex (from superior frontal gyrus), occipital cortex (from occipital gyrus), globus pallidus, putamen, and caudate nucleus.

\section{Rat studie}

\section{Animals}

Five groups of male Sprague-Dawley rats (250-300 g) were evaluated. Group $1(\mathrm{n}=8)$ consisted of normal rats. Group $2(\mathrm{n}=8)$ included rats with end-to-side portacaval shunt performed according to the guidelines of Lee and Fisher $\frac{11}{1}$; rats underwent a laparotomy, and the inferior vena cava and portal vein were isolated. The inferior vena cava was partially clamped, and an elliptical piece of vein, 1.5 times the portal vein diameter, was cut and removed. The portal vein was ligated and cut, and an end-to-side anastomosis was performed under a dissecting microscope. Total surgery time was 15 minutes. Group $3(n=8)$ included sham-operated control rats matched for weight; a laparotomy was performed and the inferior vena cava and portal vein were isolated and occluded for 15 minutes. Studies in portacaval-shunted and sham-operated rats were performed 4 weeks after surgery. Group $4(n=8)$ were rats with secondary biliary cirrhosis induced by intracholedochal injection of formalin followed by bile duct ligation as described previously. 12 These animals were studied 6 weeks after surgery. Group $5(n=8)$ consisted of rats with acute liver failure induced by a combination of portacaval anastomosis (as previously described above) followed 24 hours later by hepatic artery ligation. $\underline{13}$ Blood glucose levels were monitored and maintained at normal levels in all animals. Body temperature was monitored using a rectal thermometer probe and maintained at $37^{\circ} \mathrm{C}$ using a heat lamp if necessary. These rats were studied 15-18 hours after devascularization at coma stage of encephalopathy. All groups of rats were anesthesized with halothane throughout the surgery; postoperatively, animals were allowed to recover in individual cages under constant conditions of temperature, humidity, and light cycles, with free access to standard rat chow and water.

Rats were killed by decapitation, and brains were quickly removed and dissected with nonmetallic surgical instruments. Manganese was measured in the following brain regions: frontal cortex, globus pallidus, and caudate/putamen.

\section{Biochemisty}

Blood samples were obtained after death of rats by decapitation. The following biochemical tests were performed using routine techniques: serum bilirubin, aspartate aminotransferase, alanine aminotransferase, albumin, and alkaline phosphatase. Blood ammonia was measured using an ion-exchange method followed by a colorimetric measurement of isolated ammonia nitrogen with the Berthelot phenate-hydrochlorate reaction. 14 


\section{Portosystemic shunting}

Portosystemic shunting was evaluated in the group of rats with biliary cirrhosis. After injection of ${ }^{141}$ ceriumradiolabeled microspheres in the mesenteric vein, portosystemic shunting was calculated from the ratio between lung and liver radioactivities as described previously..$\underline{15}$

\section{Manganese}

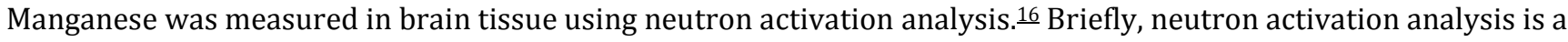
2-step technique. The first step involves the tissue being irradiated with the neutron flux of a nuclear reactor. Neutrons are then captured by the manganese nuclei and subsequently become radioactive. Once removed from the nuclear reactor, radiation is emitted as gamma rays. The second step involves detection and measurement of the energy emitted from the gamma rays. This measurement gives the total amount of the manganese in the sample and, divided by the sample weight, results in the average concentration of manganese per gram of tissue.

\section{Statistical calculations}

Results are expressed as mean \pm SEM. In human and rat studies, comparison between and within groups were made using two-way repeated-measures analysis of variance with the Tukey post hoc test. In rats, the biochemical studies were compared between groups using a one-way analysis of variance with the Tukey post hoc test. Correlations were evaluated using the Pearson's $r$ test. The level of statistical significance was set at $P<0.05$.

\section{RESULTS}

\section{Human studies}

Patient characteristics (cirrhotics and controls) are summarized in Table 1.

Table 1. Patient characteristics

\begin{tabular}{|c|c|c|c|}
\hline & Controls $(n=12)$ & Cirrhotics $(n=12)$ & \\
\hline Age (yr) & $64.3 \pm 3.7$ & $60.6 \pm 2.5$ & \\
\hline $\operatorname{Sex}(M / F)$ & $10 / 2$ & $9 / 3$ & \\
\hline \multirow[t]{7}{*}{ Diagnosis } & Respiratory failure & 3 Alcoholic cirrhosis & 7 \\
\hline & Myocardial infarction & 3 B viral cirrhosis & 3 \\
\hline & Septic shock & $2 \mathrm{C}$ viral cirrhosis & 1 \\
\hline & & \multicolumn{2}{|c|}{ Cryptogenic cirrhosis 1} \\
\hline & Lung cancer & 2 & \\
\hline & Aortic aneurysm & 1 & \\
\hline & Multiorgan failure & 1 & \\
\hline Delay between death and autopsy $(h)$ & $13 \pm 2$ & $13 \pm 4$ & \\
\hline
\end{tabular}

Groups were rigorously matched for age, sex, and time between death and sample freezing. 
As shown in Figure 1, in both controls and cirrhotics, brain manganese concentrations were higher in basal ganglia structures (pallidum, putamen, and caudate nucleus) than in cortical areas $(P<0.001)$.

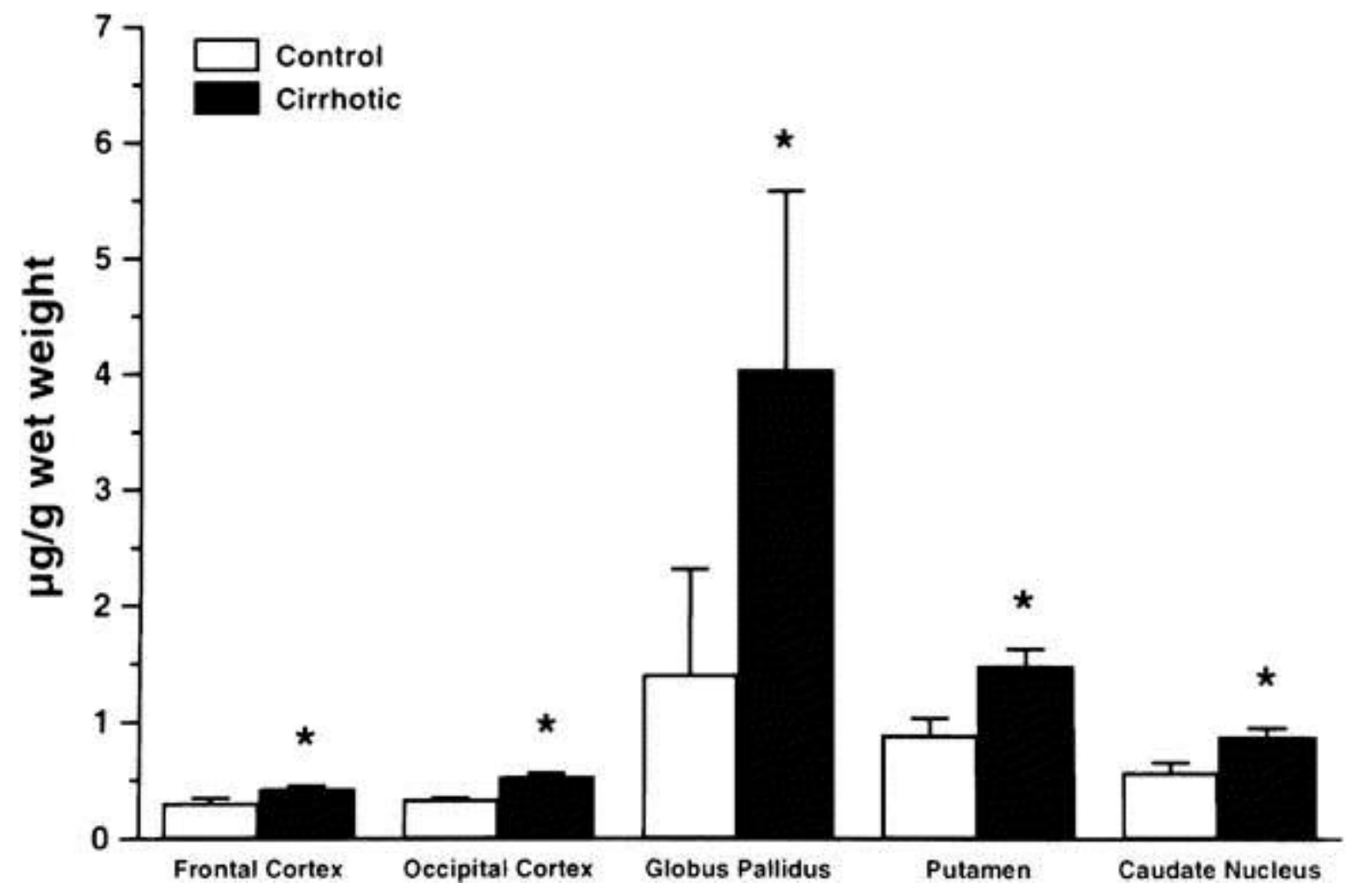

Fig. 1. Manganese concentration in postmortem human brain tissue. Data are expressed as mean \pm SEM. ${ }^{*} P<$ 0.05 , statistically different from controls.

In cirrhotics, a general increase in brain regional manganese concentration was observed compared with controls; manganese accumulation was much more pronounced in pallidum $\left(+186 \% ; 4.04 \pm 1.54 \mathrm{vs} .1 .41 \pm 0.91 \mu \mathrm{g} \cdot \mathrm{g}^{-1} \cdot\right.$ wet $\mathrm{wt}^{-1}$ in cirrhotics and controls, respectively; $\left.P<0.001\right)$ and putamen $\left(+66 \% ; 1.48 \pm 0.14 \mathrm{vs} .0 .89 \pm 0.12 \mu \mathrm{g} \cdot \mathrm{g}^{-1} \cdot\right.$ wet $\left.\mathrm{wt}^{-1} ; P<0.05\right)$, and caudate nucleus $\left(+54 \% ; 0.88 \pm 0.07 \mathrm{vs.} 0.57 \pm 0.06 \mu \mathrm{g} \cdot \mathrm{g}^{-1} \cdot\right.$ wet $\left.\mathrm{wt}^{-1} ; P<0.05\right)$. Increased brain manganese content did not correlate with patient age, etiology of cirrhosis, or history of recurrent hepatic encephalopathy (6 patients). 
Rose, C. et al., 1999. Manganese deposition in basal ganglia structures results from both portal-systemic shunting and liver dysfunction. Gastroenterology, 117(3), p.640-644.

\section{Rat studies}

Characteristics of the 5 study groups are summarized in Table 2.

Table 2. Characteristics of rat study groups

\begin{tabular}{|c|c|c|c|c|c|c|c|c|c|}
\hline & Weight $(g)$ & $\begin{array}{l}\text { Bilirubin } \\
(m g / d L)\end{array}$ & $\operatorname{AST}(I U / L)$ & $\begin{array}{l}\text { ALT } \\
(I U / L)\end{array}$ & $\operatorname{ALP}(I U / L)$ & $\operatorname{ALB}(g / L)$ & $\begin{array}{l}\text { Ammonia } \\
(\mu g / d L)\end{array}$ & $\begin{array}{l}\text { PSS } \\
(\%)\end{array}$ & $\begin{array}{l}\mathrm{PP}(m m \\
\mathrm{Hg})\end{array}$ \\
\hline $\begin{array}{l}\text { Controls }(n= \\
8)\end{array}$ & $355 \pm 25$ & $3.1 \pm 0.7$ & $155 \pm 16$ & $55 \pm 3$ & $178 \pm 21$ & $25.3 \pm 0.6$ & $181 \pm 5$ & - & - \\
\hline Sham $(n=8)$ & $356 \pm 9$ & $1.8 \pm 0.2$ & $202 \pm 28$ & $62 \pm 4$ & $191 \pm 11$ & $24.9 \pm 0.6$ & $120 \pm 4$ & - & - \\
\hline $\operatorname{PCS}(\mathrm{n}=8)$ & $281 \pm 10$ & $2.4 \pm 0.3$ & $149 \pm 13$ & $58 \pm 4$ & $241 \pm 18$ & $23.6 \pm 0.4$ & $232 \pm 16^{a}$ & - & - \\
\hline $\begin{array}{l}\text { Cirrhotics (n } \\
=8)\end{array}$ & $420 \pm 11$ & $117 \pm 11^{b}$ & $493 \pm 51^{b}$ & $90 \pm 8^{a}$ & $256 \pm 41$ & $14.5 \pm 0.7^{b}$ & $268 \pm 21^{b}$ & $57 \pm 10$ & $18.5 \pm 0.6$ \\
\hline $\operatorname{ALF}(\mathrm{n}=8)$ & $218 \pm 4$ & $39 \pm 4^{b}$ & $\begin{array}{l}15071 \pm \\
3340^{b}\end{array}$ & $\begin{array}{l}7137 \pm \\
2471^{b}\end{array}$ & $400 \pm 25^{b}$ & $25.5 \pm 0.6$ & $452 \pm 20^{b}$ & - & - \\
\hline
\end{tabular}

${ }^{a} P<0.05^{b} P<0.01$; significantly different from controls and sham groups.

NOTE. Results given as mean \pm SEM.PCS, portacaval shunt; ALF, acute liver failure (devascularization); AST, aspartate aminotransferase; ALT, alanine aminotransferase; ALP, alkaline phosphatase; ALB, albumin; PSS, portalsystemic shunting; PP, portal pressure.

Serum bilirubin levels were higher in cirrhotic rats (38-fold) and in hepatic devascularized rats (13-fold) compared with control groups of animals. Serum transaminase (aspartate and alanine) levels were also elevated in these rats, particularly in hepatic devascularized rats. Serum albumin level was significantly lower only in cirrhotic rats, with a 43\% decrease compared with controls. Alkaline phosphatase level was increased only in rats with acute liver failure with a $125 \%$ increase compared with controls. Blood ammonia was elevated in all 3 experimental liver failure groups of rats, with acute liver failure rats demonstrating the highest degree of hyperammonemia.

Portal-systemic shunting was found to be $57 \% \pm 10 \%$ in cirrhotic rats with a range from $29 \%$ to $100 \%$. Portal hypertension was found in cirrhotic rats with an average portal pressure of $18.5 \mathrm{~mm} \mathrm{Hg}$.

In all experimental groups except sham-operated animals, manganese content was higher in globus pallidus than in other brain regions $(P<0.001$; Figure 2$)$. 


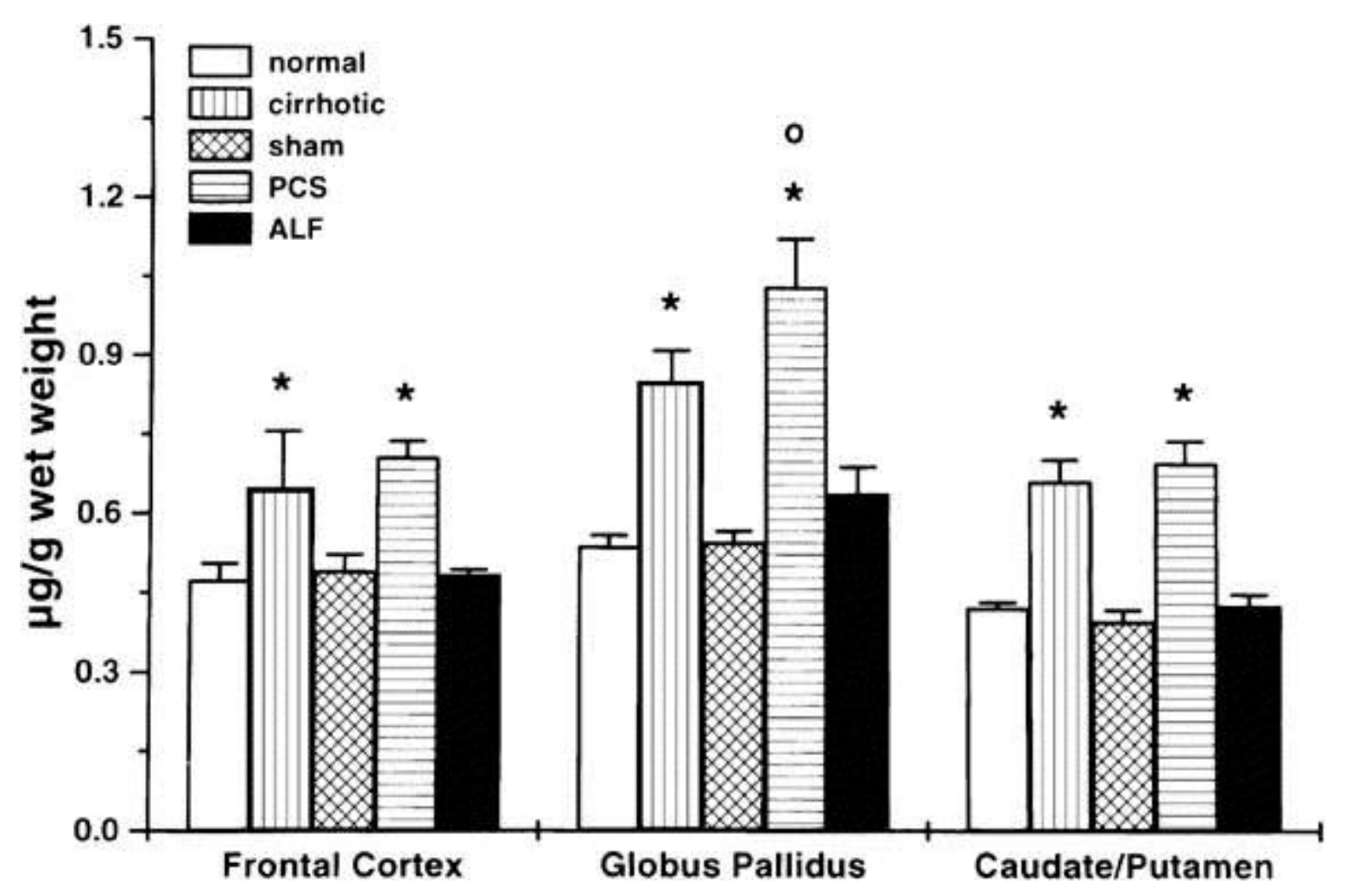

Fig. 2. Manganese concentration in rat brain tissue. Data are expressed as mean \pm SEM. ${ }^{*} P<0.05$, statistically different from controls and shams; ${ }^{\circ} P<0.05$, statistically different between portacaval-shunted (PCS) and cirrhotic rats. ALF, acute liver failure.

Brain manganese levels were higher in cirrhotic and portacaval-shunted rats than in the other 3 experimental groups $(P<0.001)$. In cirrhotic and portacaval-shunted rats, manganese content (in $\mu \mathrm{g} \cdot \mathrm{g}^{-1} \cdot \mathrm{wet}^{\mathrm{wt}} \mathrm{t}^{-1}$ ) was increased compared with normal rats in pallidum (by $27 \%$ : $0.85 \pm 0.06$ vs. $0.67 \pm 0.06, P<0.05$; by $57 \%$ : $1.05 \pm 0.07$ vs. $0.67 \pm$ $0.06, P<0.001$, respectively); in caudate/putamen (by 57\%: $0.66 \pm 0.04$ vs. $0.42 \pm 0.01, P<0.005$; by $67 \%$ : $0.70 \pm$ 0.03 vs. $0.42 \pm 0.01, P<0.001$ ); and in frontal cortex (by $41 \%: 0.65 \pm 0.11$ vs. $0.46 \pm 0.01, P<0.05$; by $47 \%: 0.68 \pm$ 0.02 vs. $0.46 \pm 0.01, P<0.05$ ). Furthermore, pallidal manganese concentrations were higher in portacaval-shunted rats than cirrhotic rats (by $23 \%$ : $1.05 \pm 0.07$ vs. $0.85 \pm 0.06 \mu \mathrm{g} \cdot \mathrm{g}^{-1} \cdot$ wet $\mathrm{wt}^{-1} ; P<0.05$ ). In contrast, no significant changes could be shown in any brain regions of rats with acute liver failure compared with control animals.

In cirrhotic rats, no significant correlation was observed between either serum albumin, serum bilirubin, serum transaminase, and alkaline phosphatase levels, blood ammonia or portal pressure, and brain manganese concentrations. However, the degree of portal-systemic shunting was correlated with pallidal manganese concentration $(r=0.72 ; P<0.05)$.

\section{DISCUSSION}

This study confirms and extends previous reports of increased pallidal manganese concentrations in cirrhotic patients. 17 and 18 The results also show that increased manganese in brain is a region-selective phenomenon with concentrations being highest in globus pallidus $>$ putamen $>$ caudate nucleus $>>$ cerebral cortex in these patients.

It has been suggested that selective manganese deposition in the pallidum could be the cause of the high signal intensity in $\mathrm{T}_{1}$-weighted images that has been consistently reported in most cirrhotic patients. $1, \underline{5}, \underline{6}, \underline{\mathrm{Z}} \underline{\underline{8}} \underline{\underline{9}} \underline{19}$ and $\underline{2} \underline{0}$ Further evidence suggesting that MR pallidal hyperintensities are best explained by manganese deposition is 
Rose, C. et al., 1999. Manganese deposition in basal ganglia structures results from both portal-systemic shunting and liver dysfunction. Gastroenterology, 117(3), p.640-644.

provided by observations in patients receiving long-term parenteral nutrition in whom typical pallidal images obtained during manganese supplementation disappeared after cessation of manganese administration. $\underline{3}$ and $\underline{4}$ Furthermore, exposure of nonhuman primates to manganese administered either by inhalation or intravenously resulted in selective pallidal hyperintensities on MRI. $\underline{21}$ and $\underline{22}$

A cause-effect relationship between chronic liver disease and region-selective brain manganese accumulation is supported by several clinical observations. For example, a patient with decompensated cirrhosis caused by the Alagille syndrome who manifested extrapyramidal symptoms and pallidal hyperintensity on MRI was found to have increased blood manganese concentrations; 2 months after liver transplantation, the extrapyramidal symptoms resolved, blood manganese level returned to normal, and pallidal MR signal hyperintensity disappeared. $\underline{23}$ Other investigators have reported a similar disappearance of pallidal MR hyperintensities several months after liver transplantation in cirrhotic patients. $\underline{7}$

Possible mechanisms responsible for manganese accumulation in the pallidum of patients with cirrhosis include a decrease in elimination via biliary excretion and an increased systemic availability due to portal-systemic shunting. Average daily oral manganese intake is $2.5-3 \mathrm{mg}$. Only $1 \%-3.5 \%$ of this normally reaches the systemic circulation because ingested manganese is rapidly cleared by the liver and excreted into the bile. $\underline{24}$ Cirrhosis is associated with a variable degree of cholestasis that can affect biliary manganese excretion. In addition, abnormalities of the microcirculation (capillarization of the sinusoids and/or intrahepatic shunting) are present in the cirrhotic liver. Alternatively (or additionally), increased blood manganese in cirrhotic patients could result from the presence of portal-systemic collaterals produced either spontaneously or after surgical portocaval anastomosis or transjugular intrahepatic portosystemic shunt.

To assess the relative importance of portal-systemic shunting and hepatobiliary dysfunction in the phenomenon of increased brain manganese in liver failure, we made use of animal models of portal-systemic shunting, of cholestasis associated with chronic liver failure, and of acute liver failure, a condition associated with neither cholestasis nor portal-systemic shunting. Both shunting and chronic cholestasis resulted in significant region-selective brain manganese accumulation.

In rats with biliary cirrhosis, there was a combination of chronic cholestasis, as evidenced by increased serum bilirubin and alkaline phosphatase levels, of liver function impairment as shown by decreased serum albumin levels, and of spontaneous shunting. All these factors potentially contribute to manganese accumulation in the brain.

Pallidal manganese concentrations were highest in shunted rats and correlated with the degree of portal-systemic shunting in cirrhotic rats, which confirms the notion that shunting is a major determinant of manganese accumulation in the brain. We observed in a previous study that blood manganese concentrations were particularly elevated in cirrhotic patients with previous portacaval shunts. $\frac{5}{}$ Furthermore, $\mathrm{T}_{1}$-weighted MR signal intensity in pallidum significantly increased after transjugular portosystemic shunt placement but not after elective sclerotherapy in similar patients. $\underline{25}$ Pallidal signal hyperintensity $\underline{26}$ on MRI was previously reported in a child with patent ductus venosus in the absence of significant liver disease.

Although evidence for a link between brain manganese deposition and MRI signal hyperintensities is convincing, a pathophysiological role for brain manganese deposition in the neurological symptoms in patients with liver disease and/or portosystemic shunts remains to be demonstrated. Manganese toxicity had previously been reported in miners after a prolonged exposure to manganese dust, resulting in extrapyramidal symptoms resembling Parkinson's disease.27 In cirrhotic patients, a high incidence of extrapyramidal symptoms, in particular rigidity, similar to that observed in Parkinson's disease, is observed when a detailed and careful neurological evaluation is performed. $\underline{5}$ and $\underline{25}$ However, recent reports evaluating the relationship between blood manganese, pallidal hyperintensity, and neurological symptoms have yielded conflicting results. $\underline{5}, \underline{6} \underline{\underline{g}}$ and $\underline{9}$ These discrepancies may result from the fact that blood manganese levels do not accurately reflect concentrations of the metal in the brain.

The final publication is available at http://dx.doi.org/10.1016/S0016-5085(99)70457-9 
Rose, C. et al., 1999. Manganese deposition in basal ganglia structures results from both portal-systemic shunting and liver dysfunction. Gastroenterology, 117(3), p.640-644.

In summary, region-selective increases in brain manganese concentration were observed in cirrhotic patients and in rats with either biliary cirrhosis or portacaval shunts. Brain manganese concentrations were particularly high in the pallidum of portacaval-shunted rats. These findings suggest that pallidal manganese accumulation observed in cirrhotic patients results from both portal-systemic shunting and impaired hepatobiliary elimination. Future studies in portacaval-shunted and cirrhotic rats could be useful in the evaluation of the potential of chelating agents to reverse manganese brain overload in chronic liver failure.

\section{REFERENCES}

1 A Pujol, J Pujol, F Graus, A Rimola, J Peri, JM Mercader, JC Garcia Pagan, J Bosch, J Rodes, E Rolosa Hyperintense globus pallidus on $\mathrm{T}_{1}$-weighted MRI in cirrhotic patients is associated with severity of liver failure Neurology, 43 (1993), pp. 65-69

2 G Pomier-Layrargues, L Spahr, RF Butterworth Increased manganese concentration in pallidum of cirrhotic patients (letter) Lancet, 345 (1995), p. 735

3 SA Mirowitz, TJ Westrich, JD Hirsh Hyperintense basal ganglia on $\mathrm{T}_{1}$-weighted MR images in patients receiving parenteral nutrition Radiology, 181 (1991), pp. 117-120

4 SA Mirowitz, TJ Westrich Basal ganglia signal intensity alterations: reversal after discontinuation of parenteral manganese administration Radiology, 185 (1992), pp. 535-536

5 L Spahr, RF Butterworth, S Fontaine, L Bui, G Therrien, P Milette, LH Lebrun, J Zayed, A Leblanc, G PomierLayrargues Increased blood manganese in cirrhotic patients: relationship to pallidal magnetic resonance signal hyperintensity and neurological symptoms Hepatology, 24 (1996), pp. 1116-1120

$6 \mathrm{~S}$ Krieger, M Jaub, 0 Jansen, L Theilman, M Geibler, D Krieger Neuropsychiatric profile and hyperintense globus pallidus on $\mathrm{T}_{1}$-weighted magnetic resonance images in liver cirrhosis

Gastroenterology, 111 (1996), pp. 147-155 7 J Kulisevsky, J Pujol, J Balanzo, C Junque, J Deus, A Capdevilla, C Villanueva Pallidal hyperintensity on magnetic resonance imaging in cirrhotic patients: clinical correlations Hepatology, 16 (1992), pp. 1382-1388

8 SD Taylor-Robinson, A Oatridge, JV Hajnal, AK Burroughs, N McIntyre, NM Desouza MR imaging of the basal ganglia in chronic liver disease: correlation of $\mathrm{T}_{1}$-weighted and magnetisation transfer contrast measurements with liver dysfunction and neuropsychiatric status Metab Brain Dis, 10 (1995), pp. 175-188

9 K Weissenborn, C Ehrenheim, A Hori, S Kubicka, MP Manns Pallidal lesions in patients with liver cirrhosis: clinical and MRI evaluation Metab Brain Dis, 10 (1995), pp. 219-228 10 SJ De Armond, M Fuscom, MM Dewey Structure of human brain: a photograph. Atlas (2nd ed)Oxford University Press, New York (1976)

11 SH Lee, B Fisher Portacaval shunt in the rat Surgery, 50 (1961), pp. 668-672

12 LA Colombato, B Rocheleau, PM Huet Intracholedochal formalin injections and bile duct ligation (FBDL) in the rat. A new model of biliary cirrhosis and portal hypertension with a normal sized biliary tree (abstr) Hepatology, 20 (1994), p. 100A

$13 \mathrm{~S}$ Websters, J Gottstein, R Levy, AT Blei Intracranial pressure waves and intracranial hypertension in rats with ischemic fulminant hepatic failure Hepatology, 14 (1991), pp. 715-720

14 SG Dienst An ion exchange method for plasma ammonia concentration J Lab Clin Med, 58 (1961), pp. 149-155

The final publication is available at http://dx.doi.org/10.1016/S0016-5085(99)70457-9 
Rose, C. et al., 1999. Manganese deposition in basal ganglia structures results from both portal-systemic shunting and liver dysfunction. Gastroenterology, 117(3), p.640-644.

15 RJ Groszmann, J Vorobioff, E Riley Splanchnic hemodynamics in portal-hypertensive rats: measurement with $\gamma$ labeled microspheres Am J Physiol, 242 (1982), pp. G156-G160

16 GG Kennedy Trace elements in polymers E Sacher, JJ Pireaux, SP Kowalczyk (Eds.), ACS Symposium Series, 8American Chemical Society, Washington, DC (1990), pp. 128-134

17 D Krieger, S Krieger, O Jansen, P Gass, L Theilmann, H Lichtnecker Manganese and chronic hepatic encephalopathy Lancet, 346 (1995), pp. 270-274

18 H Maeda, M Sato, A Yoshikawa, M Kimura, T Sonomura, M Terada, K Kishi Brain MR imaging in patients with hepatic cirrhosis: relationship between high intensity signal in basal ganglia on $\mathrm{T}_{1}$-weighted images and elemental concentrations in brain Neuroradiology, 39 (1997), pp. 550-565

19 E Inoue, S Hori, Y Narumi, M Fusita, K Kuriyama, T Kadota, C Kuroda Portal-systemic encephalopathy: presence of basal ganglia lesions with high signal intensity on MR images Radiology, 179 (1991), pp. 551-555

20 ML Zeneroli, G Cioni, G Crisi, C Vezzeli, E Ventura Globus pallidus alterations and brain atrophy in liver cirrhosis patients with encephalopathy: an MR imaging study Magn Reson Imaging, 9 (1991), pp. 295-302

21 H Shinotoh, BJ Snow, KA Hewitt, BD Pate, D Doudet, R Nugent, DP Perl, W Olanow, DB Calne MRI and PET studies of manganese intoxicated monkeys Neurology, 45 (1995), pp. 1199-1204

22 M Yamada, S Ohno, I Okayasu, R Okeda, S Hatakeyama, H Watanabe, K Ushio, H Tsukagushi Chronic manganese poisoning: a neuropathological study with determination of manganese distribution in the brain Acta Neuropathol (Berl), 70 (1986), pp. 273-278

23 AG Devenyi, TF Barron, AC Mamourian Dystonia, hyperintense basal ganglia and high whole blood manganese levels in Alagille's syndrome Gastroenterology, 106 (1994), pp. 1068-1071

24 PS Papavasiliou, ST Miller, GG Cotzias Role of liver in regulating distribution and excretion of manganese Am J Physiol, 211 (1966), pp. 211-216

25 S Krieger, M Jaub, O Jansen, A Stiehl, P Sauer, M Geibler, L Theilmann, D Krieger MRI findings in chronic hepatic encephalopathy depend on portosystemic shunt: results of a controlled prospective clinical investigation J Hepatol, 27 (1997), pp. 121-126

26 S Yanai, M Taketsugu, K Sonoda, K Condo, K Tasaki, T Hijii, J Fukushige Patent ductus venosus associated with a hyperintense globus pallidus imaging and pulmonary hypertension Eur J Pediatr, 154 (1995), pp. 526-529

27 K Nelson, J Golnick, T Korn, C Angle Manganese encephalopathy: utility of early magnetic resonance imaging Br J Intern Med, 50 (1993), pp. 510-513

The final publication is available at http://dx.doi.org/10.1016/S0016-5085(99)70457-9 